\title{
Primary Squamous Cell Carcinoma of Breast in a Young Female: An Institutional Experience with Review of Literature
}

Abhishek Purkayastha ${ }^{1 *}$, Sankalp Singh ${ }^{1}$, Niharika Bisht ${ }^{1}$, Divya Shelly ${ }^{2}$, Reena Bharadwaj ${ }^{2}$, Harinder Pal Singh ${ }^{3}$, Amul Kapoor $^{3}$, Deepak Mulajkar $^{3}$, Sameer Gupta ${ }^{4}$ and Richa Joshi ${ }^{4}$

${ }^{1}$ Department of Radiation Oncology, Command Hospital (Southern Command), Pune, India

${ }^{2}$ Department of Onco-Pathology and Molecular Science, Armed Forces Medical College, Pune, India

${ }^{3}$ Department of Medical Oncology, Command Hospital (Southern Command), Pune, India

${ }^{4}$ Department of Surgical Oncology, Command Hospital (Southern Command), Pune, India

*Corresponding author: Abhishek Purkayastha, Department of Radiation Oncology, Command Hospital (Southern Command), Pune, India, Tel: 9650901736; E-mail: abhi5296@gmail.com

Received date: January 17, 2018; Accepted date: January 25, 2018; Published date: February 16, 2018

Copyright: ( 2018 Abhishek P, et al. This is an open-access article distributed under the terms of the Creative Commons Attribution License, which permits unrestricted use, distribution, and reproduction in any medium, provided the original author and source are credited.

\begin{abstract}
Invasive or infiltrating ductal carcinoma is the most common type of breast cancer while primary squamous cell carcinoma of breast is extremely rare occurring in $0.04 \%-0.1 \%$ of all breast cancer cases known for aggressive behaviour and poor prognosis. We hereby report a case of primary squamous cell carcinoma of breast in a 31-yearold female who presented with a left breast lump of 2 months duration. Lumpectomy done at another non-oncology centre showed invasive ductal carcinoma with margin positivity for which she underwent modified radical mastectomy at our institute. Post-operative immune-histopathology revealed triple negative squamous cell carcinoma. Her metastatic work-up with whole-body positron emission tomography scan was negative. She was treated with adjuvant chemotherapy doxorubicin, cyclophosphamide and docetaxel followed by loco-regional radiotherapy to left chest wall which she tolerated well. No hormonal therapy was given in view of hormone receptor negativity. She is presently on follow-up for more than 6 months without any evidence of recurrence or distant metastasis. This case is being presented to highlight its extreme rarity, it's occurrence in a young female, the diagnostic and therapeutic challenges it presented and the overall prognosis of this neoplasm.
\end{abstract}

Keywords Primary; Squamous cell carcinoma; Breast; Young; Female

Abbreviations SqCC: squamous cell carcinoma; IDC: invasive ductal carcinomas; RT: radiotherapy; BIRADS: Breast Imaging Reporting and Data System; HPR: histopathology report; IHC: immunohistochemistry; PET: positron emission tomography; MRM: modified radical mastectomy; ER: estrogen receptor; PR: progesterone receptor; Her-2/Neu: human epidermal growth factor receptor-2; EGFR: epidermal growth factor receptor; BCS: breast conservation surgery; CT: computed tomography; MRI: magnetic res $\neg$ onance imaging; OS: overall survival; RFS: relapse free survival; DFS: disease free survival.

\section{Introduction}

Primary SqCC of the breast is diagnosed when squamous component comprises more than $90 \%$ of the malignant cells [1]. SqCC of the breast is an extremely rare neoplasm accounting for only $0.04 \%-0.1 \%$ of all breast tumors [2]. This variety is highly aggressive with more incidence of loco-regional recurrence, distant dissemination and treatment failures associated with a poor prognosis as compared to IDC [3]. With only solitary case reports and few case series without any randomized controlled trials in world literature, and management protocols are extrapolated from treatment algorithms of IDC. Upfront mastectomy followed by chemotherapy and or RT with addition of hormonal therapy in few selected cases of hormone receptor positivity is the current strategy being followed worldwide. As the aetiology and pathogenesis of this unusual entity being still unclear [4] there are still no universally accepted guidelines for its diagnosis and treatment approach, thus posing a diagnostic and therapeutic dilemma for treating oncologists.

\section{Case Report}

A 31-year-old female with no known co-morbidity presented with a history of lump left breast of 2 months duration. The lump was gradually progressive in size, not associated with pain, nipple discharge and retraction or dimpling of the skin. There was no family history of breast malignancy. Physical examination revealed a firm, non-tender and mobile mass of approximately $4 \times 3 \mathrm{~cm}$ in the infero-lateral quadrant of left breast. The skin and the nipple-areola complex were not involved. No enlarged axillary lymph nodes were noted on ipsilateral side. Right breast was normal. Sono-Mammography (Figure 1) showed a $40 \times 33 \mathrm{~mm}$ well-defined hypoechoic solid irregular mass in left infero-lateral quadrant with obscured margins without any microcalcification or other associated abnormalities. Margins invasive at places and smoothly lobulated at other places suggestive of BIRADS III. She was advised biopsy from the left breast lump for a definite diagnosis. 
Citation: Abhishek Purkayastha, Sankalp Singh, Niharika Bisht, Divya Shelly, Reena Bharadwaj, et al. (2018) Primary Squamous Cell Carcinoma of Breast in a Young Female: An Institutional Experience with Review of Literature. J Integr Oncol 7: 204. doi: $10.4172 / 2329-6771.1000204$

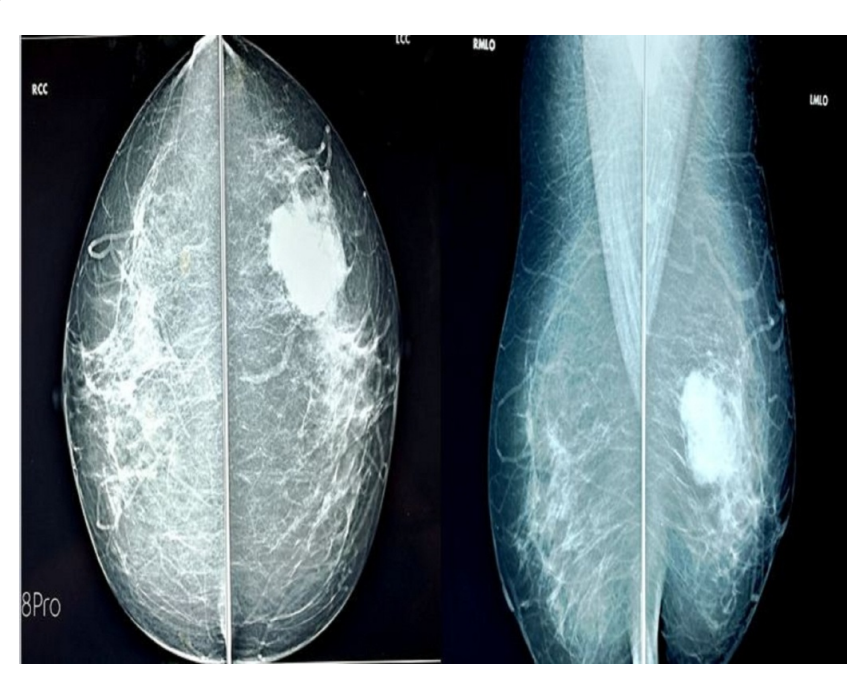

Figure 1: Sono-mammography of left breast showing well-defined hypoechoic solid irregular mass in left infero-lateral quadrant with obscured margins without any microcalcification or other associated abnormalities.

However she presented to us after 12 weeks undergoing lumpectomy of left breast without any axillary clearance from a nononcology centre. HPR of the lumpectomy specimen from the other centre showed IDC grade-III with margin positivity for which she underwent left MRM along with left axillary lymphadenectomy at our centre. Post-operative HPR (Figure 2) reported by onco-pathologist at our centre however revealed a tumour composed of sheets and clusters of round to polyhedral cells with pleomorphic hyperchromatic nuclei, prominent nucleoli and a moderate amount of eosinophilic cytoplasm. Individual cell keratinization was also noted. Multinucleated tumour cells and brisk mitoses were seen. At places, cystic spaces with central areas of necrosis and lining of the similar tumour cells were also noted. No areas of adenocarcinoma were seen. No chondroid, osseous or mesenchymal differentiation was seen. A total of 17 axillary lymphnodes were dissected, all were free of any tumor deposits. On IHC, the tumour cells were positive for CK5/6 and p63 (Figure 3); while were negative for estrogen and progesterone receptors and Her2neu (Figure 4). Since the overlying skin and nipple areola complex were free of tumour and metastatic work-up with PET scan was negaative, hence the diagnosis of primary SqCC of the breast was rendered. She received adjuvant chemotherapy 4 cycles of doxorubicin and cyclophosphamide (AC) followed by 4 cycles of docetaxel. The patient was further treated with loco-regional RT to left chest wall to a conventional dosage schedule of 50 Gy in 25 fractions 5 days a week which she tolerated well. No hormonal therapy was given in view of triple negative disease. Presently she is on close follow-up for more than 6 months without any evidence of local or distant faluire.

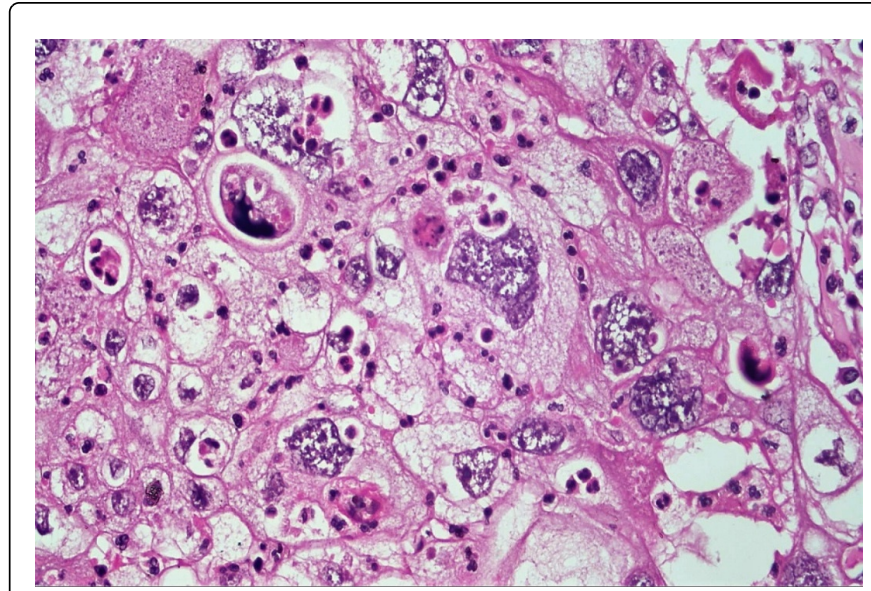

Figure 2: Post-MRM HPR showing a tumour composed of sheets and clusters of round to polyhedral cells with pleomorphic hyperchromatic nuclei, prominent nucleoli and a moderate amount of eosinophilic cytoplasm. Individual cell keratinization was also noted. No areas of adenocarcinoma were seen (H \& E 100 X).

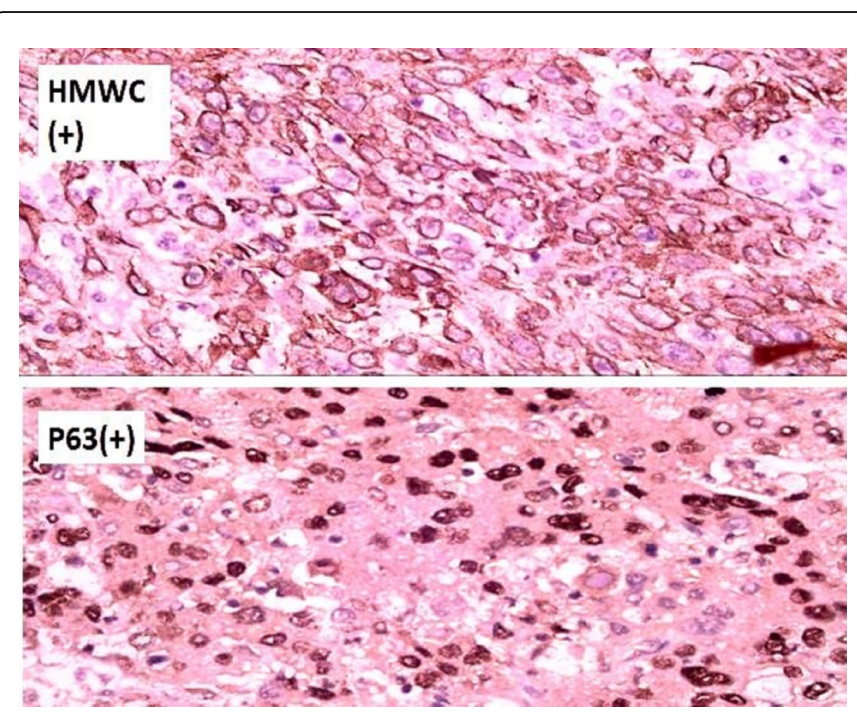

Figure 3: IHC of the tumour cells showing positivity for keratin $5 / 6$ (CK5/6) and p63 (100 X). 
Citation: $\quad$ Abhishek Purkayastha, Sankalp Singh, Niharika Bisht, Divya Shelly, Reena Bharadwaj, et al. (2018) Primary Squamous Cell Carcinoma of Breast in a Young Female: An Institutional Experience with Review of Literature. J Integr Oncol 7: 204. doi: $10.4172 / 2329-6771.1000204$

Page 3 of 4

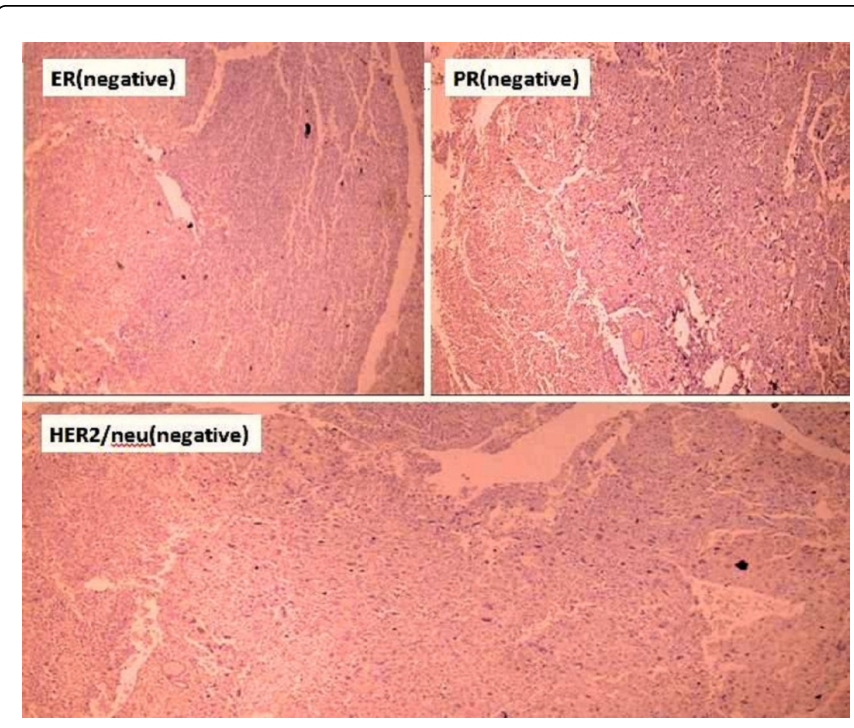

Figure 4: IHC staining negative for estrogen and progesterone receptors and Her2neu (100 X).

\section{Discussion}

SqCC is known to be primarily a malignancy of the skin, head and neck, esophagus, cervix and anal canal, but its occurrence as a breast primary is highly unusual. It accounts for a mere $0.04 \%-0.1 \%$ of all breast tumors as per world literature [2]. The treatment cards and case summary of patients who had received treatment at our tertiary care cancer institute over the last 10 years from January 2007 to December 2017 were perused and retrieved. However, only one case of primary SqCC breast out of 490 breast cancer cases was found. Troell [4,5] in 1908 first described the existence of an entity called SqCC of breast. Regarding its origin, several theories do exist like evolution from focus of squamous metaplasia within a pre-existing adenocarcinoma of the breast or arise directly from basal progenitor cells of mammary duct epithelium [6] and maybe this can be the reason why initial HPR showed IDC component in our case. The diagnosis of primary SqCC of the breast is made in the absence of another primary SqCC in a second site and absence of any skin involvement $[7,8]$. SqCC breast has been classified as primary or metaplastic with $100 \%$ and $50 \%$ of the malignant cells showing squamous component respectively [9]. However, cells with $>90 \%$ squamous morphology have also been considered as a primary SqCC [10]. Not only adenocarcinoma or duct epithelium, SqCC can also arise from benign lesions like a breast cyst, abscess or mastitis [11] and even from capsules around silicone breast implants [12].

Regarding hormone receptors, estrogen (ER) and progesterone receptors (PR) have been found to be negative in more than $90 \%$ of the cases of primary SqCC [13] along with $\mathrm{Her}-2 / \mathrm{Nu}$ [14]. However, Her-2/Nu overexpression has been reported [15] and also BRCA-1 positivity has been documented in literature [16], but both were negative in our case. Expression of EGFR in primary SqCC of breast has also been reported [17-19] which gives the scope of including targeted therapy in the treatment regimens. Clinically differentiating primary SqCC breast from other breast pathologies is difficult. It is mostly noticed as a firm to hard nodular palpable mass with a rapid growth usually presenting over few weeks to few months [13].
Although tumor size at diagnosis has been found to be more than $4 \mathrm{~cm}$ in most cases of SqCC breast compared to IDC [20], however its implication in the overall prognosis is not known yet. However, axillary lymph node involvement of $10-15 \%$ has been reported with larger tumors resulting in poor prognosis [21]. Axillary lymph node involvement in SqCC has been documented to be less frequent than adenocarcinoma [22]. Breast SqCC has been considered to be a malignancy of older age group [14] with the average age at diagnosis being 52 years [7], however our patient was a young female of 31 years.

The definite diagnosis of breast SqCC is challenging as typical clinical manifestations, CT scan and MRI is generally not sufficient to establish a diagnosis. Mammography usually shows no typical findings apart from the lack of microcalcifications and cystic appearance in about $50 \%$ of the cases [23]. Ultra-sonogram of the breasts may show hypoechogenic mass with cystic components, thus being more helpful in differentiating SqCC [24]. Adequate tissue specimen with immunohistopathological diagnosis remains the gold standard [17]. The role of PET scan as diagnostic modality remains an area of further investigation [25], although it was used in our case to rule out any other primary in the body. The management of SqCC breast has always been a challenge for oncologists with no definite treatment guidelines. Upfront quadrantectomy [21] or mastectomy [22] with axillary lymph node dissection or mastectomy with sentinel lymph node biopsy [26] has been considered the most favourable therapeutic option due to the invasiveness and dismal prognosis. While BCS has not been recommended in few reports [27], others have advocated BCS as a safe and effective option for local control $[17,28,29]$.

Chemotherapy regimens used for IDC have been reported to be of no effect in a primary SqCC breast [30], however due to lack of any established systemic therapy regimen we used adjuvant $\mathrm{AC}$ followed by taxane. Few authors have followed the treatment guidelines for a squamous cell line and have reported effectiveness of platinum based chemotherapy in SqCC breast $[18,31]$ while neoadjuvant chemotherapy has been found to be of no benefit [24]. Due to the triple negative nature in majority of SqCC cases, endocrine therapy and anti-HER2 targeted therapy is generally not utilized. Overexpression of EGFR in SqCC breast encourages the possibility of using anti-EGFR targeted therapy with Cetuximab or Gefitinib [18]. Regarding the role of post-operative RT, there are no concrete reports regarding its effectiveness in preventing loco-regional relapse or enhancing OS in a primary SqCC breast as cases have been reported to recur at irradiated field [24], thus being labelled as a radioresistant tumor [25]. We used a dose of $50 \mathrm{~Gy}$ in 25 fractions at a rate of $2 \mathrm{~Gy}$ per fraction following the standard radiation protocol. Early initiation of RT immediately following adjuvant chemotherapy have been reported to improve loco-regional relapse with relapse-free rate of $45 \%$ among those receiving and 33\% among those not receiving adjuvant RT [27]. The 5-year OS has been around 67\% [14], 44\% in patients treated with adjuvant RT and $33 \%$ in patients not receiving RT [27]. The 5 -year RFS has been reported to be about $62 \%$ for patients treated with adjuvant RT and 45\% for non-RT patients [27].

\section{Conclusion}

Primary SqCC of breast is an extremely rare and aggressive neoplasm associated with frequent loco-regional relapses and distant dissemination resulting in increased incidence of morbidity and mortality. By reporting this unusual case experienced by us, we recommend that the diagnosis of primary SqCC of breast should always be considered in patients presenting with a breast lump with 
Citation: Abhishek Purkayastha, Sankalp Singh, Niharika Bisht, Divya Shelly, Reena Bharadwaj, et al. (2018) Primary Squamous Cell Carcinoma of Breast in a Young Female: An Institutional Experience with Review of Literature. J Integr Oncol 7: 204. doi: $10.4172 / 2329-6771.1000204$

Page 4 of 4

supporting IHC differentiating it from other common breast pathologies so as to initiate an appropriate management strategy. Presently there are no definite treatment guidelines for this disease per se as most information is based on solitary case reports and small case series. Also a better understanding and interpretation of the molecular and biological mechanism of the disease process may help to device therapeutic strategies to manage this entity. The role of EGFR and other signalling pathways in this disease needs to be investigated further to establish their role as a potential treatment target. More sophisticated diagnostic techniques, gene profiling, novel targeted regimens, optimal RT dosages should be devised which can improve the DFS, OS and RFS of the patients.

\section{Acknowledgement}

We thank the patient and her relatives for allowing us to publish her case.

We also like to thank department of Radiology and Nuclear Medicine, Command Hospital (Southern Command), Pune, India.

The manuscript has been read and approved by all the authors, the requirements for authorship have been met, and each author believes that the manuscript represents honest work.

\section{References}

1. Rosen PR (2001) Rosen's Breast Pathology. Philadelphia, PA: Lippincott Williams \& Wilkins 455-461.

2. Gupta C, Malani AK, Weigand RT, Rangineni G (2006) Pure primary squamous cell carcinoma of the breast: A rare presentation and clinicopathologic comparison with usual ductal carcinoma of the breast. Pathol Res Pract 202: 465-469.

3. Mitra B, Pal M, Debnath S, Paul B, Saha TN, et al. (2011) Primary squamous cell carcinoma of breast with ipsilateral axillary lymph node metastasis: An unusual case. Int J Surg Case Rep 2: 194-197.

4. Bhosale SJ, Kshirsagar AY, Deshmukh SJ, Jagtap SV, Langade YB (2013) Squamous cell carcinoma of the breast. Am J Case Rep 14: 188-190.

5. Troell A (1908) Zwei Falle von Palttenepithelcarcinom. Nord Med Ark 1: 1-11.

6. Farrand R, Lavigne R, Lokich J, McAuley R, Sparling S, et al (1979) Epidermoid carcinoma of the Breast. J Surg Oncol 12: 207-212.

7. Weigel RJ, Ikeda DM, Nowels KW (1996) Primary squamous cell carcinoma of the breast. South Med J 89: 511-515.

8. Sheen-Chen S, Chen Y, Chou FF, Eng HL (1992) Primary squamous cell carcinoma of the breast. South Med J 85: 207-209.

9. Honda M, Saji S, Horiguchi S, Suzuki E, Aruga T, et al. (2011) Clinicopathological analysis of ten patients with metaplastic squamous cell carcinoma of the breast. Surg Today 41: 328-332.

10. Porzio R, Cordini C, Orsi N, Brigati F, Paties CT, et al. (2014) Primary squamous cell carcinoma of the breast after cured bilateral breast cancer. In Vivo 28: 1155-1158.

11. Murialdo R, Boy D, Musizzano Y, Tixi L, Ballestrero A, et al. (2009) Squamous cell carcinoma of the breast: a case report. Cases J 2: 7336.

12. Kitchen SB, Paletta CE, Shehadi SI, Bauer WC (1994) Epithelialization of the lining of a breast implant capsule. Possible origins of squamous cell carcinoma associated with a breast implant capsule. Cancer 73: 1449-1452.

13. Siegelmann-Danieli N, Murphy TJ, Meschter SC, Stein M.E, Prichard J (2005) Primary squamous cell carcinoma of the breast. Clinical of breast cancer 6: 270-272.

14. Behranwala KA, Nasiri N, Abdullah N, Trott PA, Gui GPH (2003) Squamous cell carcinoma of the breast: clinico-pathologic implications and outcome. Eur J Surg Oncol 29: 386-389.

15. Karamouzis MV, Fida A, Apostolikas N, Rigatos G (2006) A case of Her-2 positive squamous cell breast carcinoma: an unusual presentation of an unusual clinical entity. Eur J Surg Oncol 32: 1250-1251.

16. Breuer A, Kandel M, Fisseler EA, Sutter C, Schwaab E, et al. (2007) BRCA-1 germline mutation in a women with metaplastic squamous cell breast cancer. Onkologie 30: 316-318.

17. Chen XL, Luo J, Xu FL (2016) Squamous cell carcinoma of the breast: particularity and clinical management. Int J Clin Exp Med 9: 14167-14174.

18. Wang J, Zhang X, He J, Yang M, Tang J, et al. (2014) Co-expression of EGFR and CK5/6 in primary squamous cell carcinoma of the breast. Med Oncol 31: 172 .

19. Kimura F, Iwaya K, Kawaguchi T, Kaise H, Yamada K, et al. (2010) Epidermal growth factor-dependent enhancement of invasiveness of squamous cell carcinoma of the breast. Cancer Sci 101: 1133-1140.

20. Gupta N, Vashisht R, Nimbran V, Gupta R, Dhingra N, et al. (2012) Primary squamous cell carcinoma of the breast: case report and management decisions. J Cancer Res Ther 8: 323-325.

21. Punzo C, Fortarezza F, De Ruvo V, Minafra M, Laforgia R, et al. (2017) Primitive squamous cell carcinoma of the breast (SCCB): case report of an uncommon variant of metaplastic carcinoma. G Chir 38: 139-142.

22. Menes T, Schachter J, Morgenstern S (2003) Primary squamous cell carcinoma (SqCC) of the breast. AM J Clin Oncol 26: 571-573.

23. Zoltan B, Lawrence K, Coleman J (2001) Pure squamous cell carcinoma of the breast in a patient with previous adenocarcinoma of the breast: a case report and review of the literature. Am Surg 67: 671-673.

24. Flikweert ER, Hofstee M, Liem M (2008) Squamous cell carcinoma of the breast: a case report. World J Surg Oncol 6: 135.

25. Healy CF, Feeley L, Leen E, Walsh TN (2006) Primary squamous cell carcinoma of the breast: value of positron emission tomography scanning in confirming the diagnosis. Clin Breast Cancer 5: 413-415.

26. Lim GH, Acosta HA, Gudi MA (2017) Natural history of metaplastic squamous cell breast cancer: a case report and literature review on surgical management. Gland Surg 6: 738-741.

27. Hennessy BT, Krishnamurthy S, Giordano S, Buchholz TA, Kau SW, et al. (2005) Squamous cell carcinoma of the breast. J Clin Oncol 23: 7827-7835.

28. Carbone S, Lobo AR, Lamacchia A, Almenar GA, Martin Hernandez R, et al. (2012) Primary squamous cell carcinoma of the breast: A rare case report. Rep Pract Oncol Radiother 17: 363-366.

29. Zhang X, Zhang B, Zang F, Zhao L, Yuan Z, et al. (2016) Clinical features and treatment of squamous cell carcinoma of the breast. Onco targets and Therapy 9: 3181-3185.

30. Rostock RA, Bauer TW, Eggleston JC (1984) Primary squamous carcinoma of the breast: A review Breast 10: 27-31.

31. Tsung SH (2012) Primary pure squamous cell carcinoma of the breast might be sensitive to Cisplatin-based chemotherapy. Case Rep Oncol 5: 561-565. 